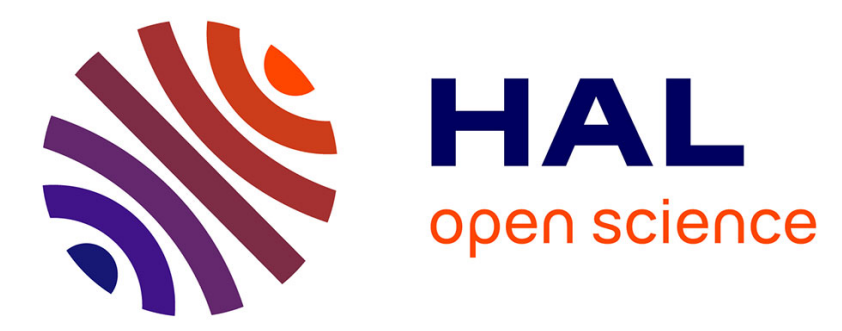

\title{
Early Index for Detection of Pediatric Emergency Department Crowding
}

\author{
Guillaume Bouleux, Eric Marcon, Olivier Mory
}

\section{To cite this version:}

Guillaume Bouleux, Eric Marcon, Olivier Mory. Early Index for Detection of Pediatric Emergency Department Crowding. IEEE Journal of Biomedical and Health Informatics, 2015, 19 (6), pp.19291936. 10.1109/JBHI.2014.2350996 . hal-01359977

\section{HAL Id: hal-01359977 https://hal.science/hal-01359977}

Submitted on 5 Sep 2016

HAL is a multi-disciplinary open access archive for the deposit and dissemination of scientific research documents, whether they are published or not. The documents may come from teaching and research institutions in France or abroad, or from public or private research centers.
L'archive ouverte pluridisciplinaire HAL, est destinée au dépôt et à la diffusion de documents scientifiques de niveau recherche, publiés ou non, émanant des établissements d'enseignement et de recherche français ou étrangers, des laboratoires publics ou privés. 


\title{
Early Index for Detection of Pediatric Emergency Department Crowding
}

\author{
Guillaume Bouleux*, Eric Marcon*, Olivier Mory ${ }^{\dagger}$
}

\begin{abstract}
When epidemics occur, such as is the case for Bronchiolitis in pediatric ED, the patient flow in the ED incontestably increases and can lead to crowding. We bypassed this difficulty of forecasting patient flow with aggregated weekly or monthly data, by tackling the problem from a different point of view. We used daily data to build a multi-period Serfling-based model. This model is hereinafter assimilated to normal ED flow. We then used the fourth-order moment of distribution of the Time Series, obtained from the difference between the estimated model and the real data, to provide an early index announcing abnormal ED patient flow. This index is parameterdependent and we provide a criterion to assist in selecting the optimal parameters. A simple program based on this methodology has been developed and has been given to the pediatric physicians for testing. Thanks to this index, the Pediatric ED was able to anticipate crowding almost three weeks before the height of the Bronchiolitis epidemic.
\end{abstract}

\section{Index Terms}

Crowding Detection, Modeling methodologies, Statistical computing, Time series analysis

\section{INTRODUCTION}

Although the literature is abundant regarding the effects of Emergency Department (ED) breakdown in North America, the same effects are observed in Europe, and particularly in France. It also seems that the literature is very vague when defining these effects. So, for clarity, we propose to adopt the definition given in [1], which characterizes ED breakdown as "crowding". The definition of crowding, originally proposed by the American College of Emergency Physicians, is: "Crowding occurs when the identified need for emergency services exceeds available resources for patient care in the emergency department, hospital, or both". From this definition, we interpreted crowding as a phenomenon that involves the interaction between supply and demand. Each hospital department has a specific capacity, so the problem of crowding instantly has a knock-on effect in terms of organization. Over the past few decades, much research has looked into ways of solving these organizational problems. One way is to assimilate Hospitals to classical companies in which the patients are considered as products. This dematerializes patients and does not take into account all patient constraints, but it helps to model and solve the problem. For example, discrete event-based modeling [2], [3], [4], like ARENA, provides good solutions for reducing patient waiting times, improving scheduling or minimizing the number of rooms. Using this kind of approach to deal with the problem enables solutions to complex situations, in which many parameters interact potentially non-linearly, to be found. However, patient flow is an essential parameter, and when modeled using exponential or equivalent laws it does not reflect reality. This implicitly suggests that knowing the patient flow could really help in developing solutions. Furthermore, patient flow is the cause and the origin of the effects of crowding. This underlying idea was very recently introduced by [5] or [6]; the latter refers to the previous work by [7], in which ED patient flow was studied to predict Inpatient Unit (IU) beds.

The organizational problem in Hospitals can be reduced to the study of ED patient flow; this point has been illustrated by [8] and reinforced by recent French studies. It appeared that in French Teaching hospitals (i.e. French $\mathrm{CHU}$ ), and particularly Pediatric ED, Bronchiolitis and Diarrhea were the two main diseases (epidemics) which

\footnotetext{
${ }^{*}$ University of Lyon, University of Saint Etienne, DISP, IUT Roanne, France. Tel.: +33477448152, Fax: +33477448951, \{guillaume.bouleux,marcon\}@univ-st-etienne.fr

${ }^{\dagger}$ Urgences Medico-Chirurgicales Pediatriques (Pediatric ED), Teaching Hospital (CHU) Saint Etienne, 42055 Saint Etienne CEDEX 2 , France, Tel: +33477828632, Fax: +33477828098, omory@chu-st-etienne.fr
} 
caused the most disruption as a consequence of crowding (see Fig.1 for confirmation). The ratio between the number of inpatient beds and the number of patients arriving at the ED with Bronchiolitis (i.e. Bronchiolitis ED flow) was around 34\%. The data collected from Saint Etienne Hospital concerning inpatient stays showed that the average Length of Stay (LoS) was around 4 days. Consequently, even if a third of Bronchiolitis patients became inpatients, the cumulative effect yielded an insufficient number of beds resulting in crowding. We do stress once again that crowding results from patient flow. It is more difficult to focus on bed occupancy as it is a complex mix of patient flow and organizational strategies.

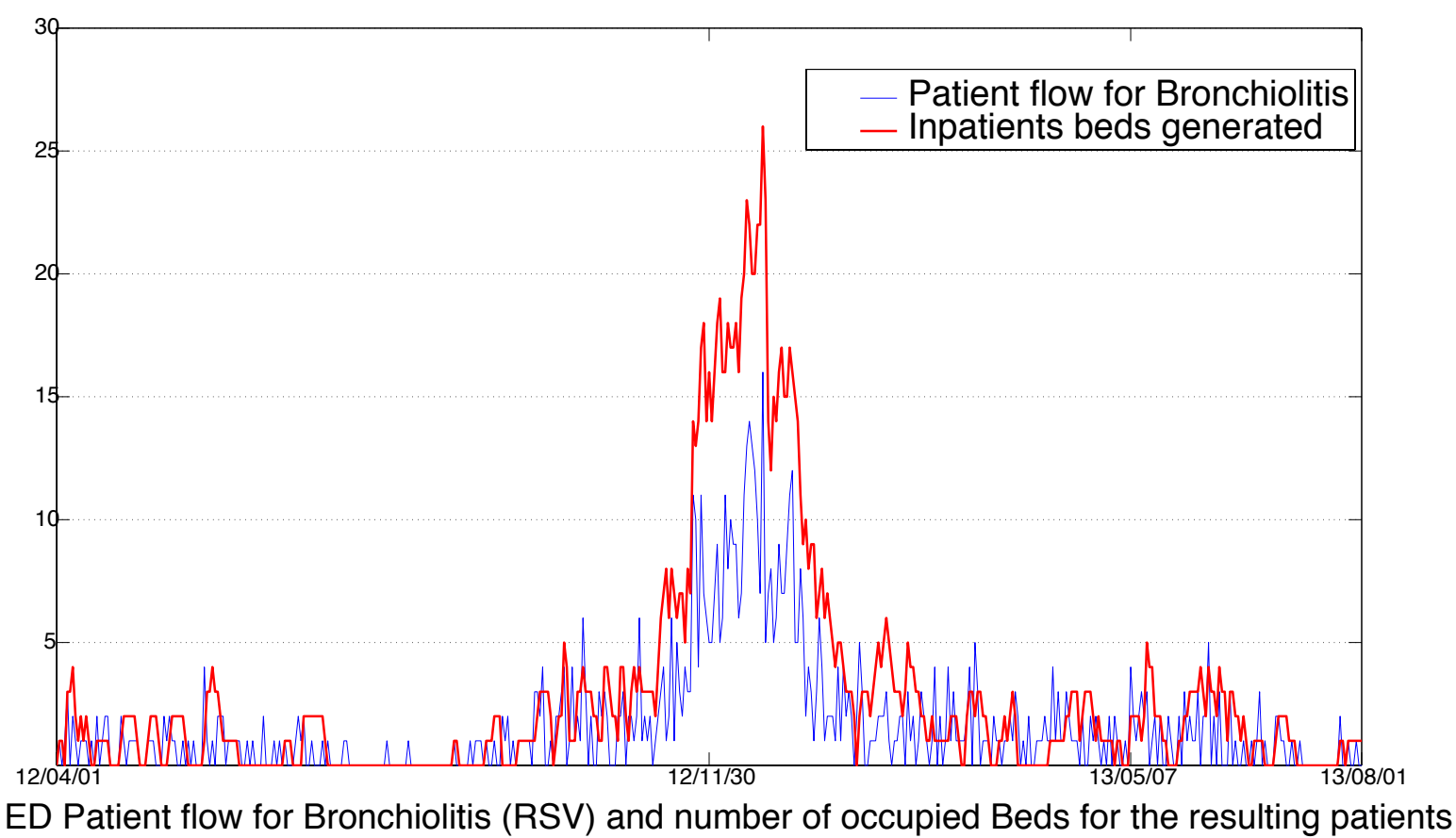

Pediatric ED Patient flow for Bronchiolitis (RSV) and number of occupied Beds for the resulting patients

Fig. 1. One-year comparison between the flow of patients diagnosed with Bronchiolitis in the Pediatric ED and the number of inpatient beds generated. Source: Saint Etienne Teaching Hospital (CHU Saint Etienne), France

The strategy adopted by Saint Etienne (France) Pediatric ED to combat crowding was to request an action plan at a fixed period, usually mid-January. The request involves increasing the number of nurses on-call, freeing up weekday beds for the weekend, and adding 7 extra beds in the 0-6 year-old Inpatient Unit (IU) to increase the bed capacity. Finally, in the case of insurmountable epidemics, inpatients can be transferred to other public or teaching hospitals. This was the case for Saint Etienne Hospital in 2012. All these decisions were made without the relevant, quantitative information relating to when the crowding would occur. The action plan was only activated mid-January, at the time of the official request, due to employee vacations or days off due to outstanding leave. Incidentally, this organizational problem has been circumvented by forcing all the practitioners to take any remaining leave before December.

Now let us put things into context. Between December and February, physicians and ED practitioners clearly knew they would have to cope with an abnormal workload as a consequence of what [9] and [10] characterize as cyclic (almost periodic) epidemics. We consider that forcing all the practitioners to be ready for that period is an empirical decision to help cope with what we define as normal activity, since it is typical to have more cases of Bronchiolitis and Diarrhea during this period. The mid-January emergency plan thus deals with any abnormal activity, which can be assimilated to crowding. However, the study by [11] shows that the dates when epidemics occur vary in time and amplitude, yielding non-stationary properties, as illustrated in Fig.2. The number of Pediatric ED patients diagnosed with Bronchiolitis between 1st January 2011 and the end of December 2013 are reported. The Bronchiolitis epidemics appeared in early February 2011, mid-November 2012, and early December 2013 and 2014, and were of varying amplitude and duration, indicating that abnormal activity can occur at the start of winter 
as well as at the very end. So, we viewed the problem in the following way: instead of trying to predict Pediatric ED flow using SARIMA or ARIMA-based methods (see for instance the work by [12], [13], [14]), or the main subset related to Bronchiolitis and Diarrhea, we proposed an early index to predict abnormal ED flow. A sufficiently early index should leave the ED or Hospital enough time to find organizational solutions and thus minimize the effects of crowding. The underlying idea has already been proposed by [15], [16], [17], and a few decades ago by [18], for influenza. The model and the early index given by Serfling were developed with respect to aggregated weekly or monthly data, yet to improve decision-making and Hospital re-organization, data collected daily should be processed. The Serfling index for detecting the onset of an epidemic is inadequate for this kind of data. We propose to overcome this by using a Serfling Pediatric ED flow based-model in conjunction with the fourth-order moment of distribution of a Time Series obtained from the difference between the model and the real data. In this way, we can provide a model for normal Bronchiolitis ED flow and early detect when abnormal conditions will appear early.

The Serfling Bronchiolitis ED flow model is presented in section II. In section III we discuss the idea based on the Fourth-order moment of ED flow distribution and section IV looks at the problem of optimal parameter choice. The methodology and its importance for Saint Etienne Pediatric ED is illustrated in section V and finally a conclusion is drawn in section VI.

The capital and boldface letters are assimilated to matrices, boldface letters stand for vectors, and scalars are represented either by classical letters or by the row-column subscript. Finally, all hat quantities are assimilated to estimates.

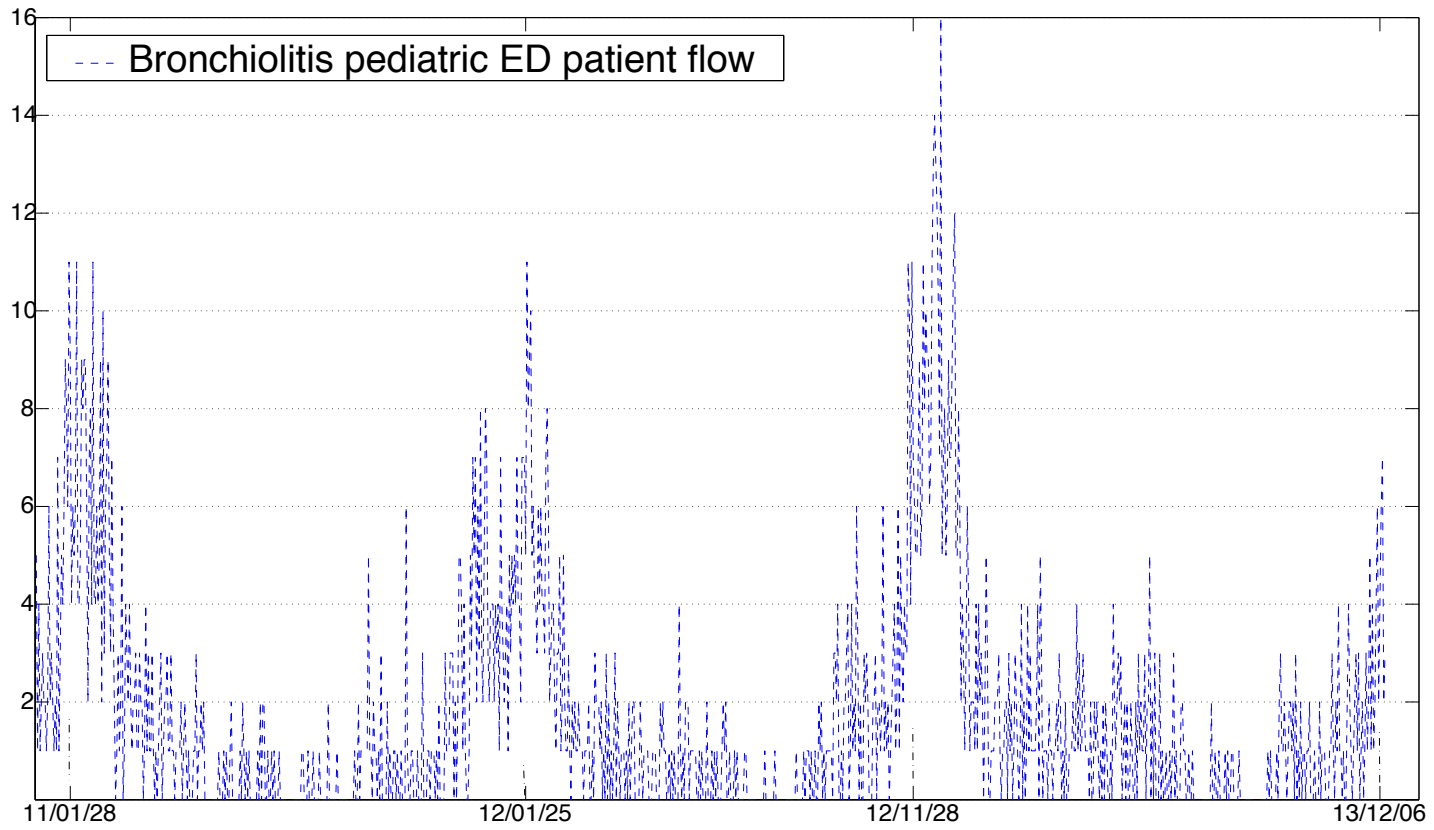

Fig. 2. Saint Etienne Teaching Hospital (CHU Saint Etienne), France, ED flow of patients diagnosed with Bronchiolitis for the period of 1st January 2011 to 12 th December 2013

\section{MODELING OF NORMAL ACTIVITY}

Research dealing with the problem of crowding effects in ED has been ongoing for many years. Attempts to minimize crowding have focused mainly on trying to predict the future, i.e. forecasting. At this time, the topic is so vast that it would be easy to feel extremely lost. [19] and [20] reviewed some forecasting methods. Yet, although they are recent, Bayesian estimation based on classical triage was not mentioned. We invite the reader to look at the dissertation by [21] and the related references for a better understanding of how Bayesian methods 
can be useful in short-term forecasting of crowding. Neural networks are only cited in [19], but their kernel-based extension does not appear to be essential as it is totally absent. The work by [22] fills this gap with a comparison between SARIMA predictive models and SVM (Support Vector Machines). Finally, most of the forecasting methods addressed previously use the notion of Time Series representation of ED flow, so most of them only use data collected over time for forecasting. Other methods exist which introduce temperature and calendar variables, such as in [23], or diffusion and contact time variables as in [4]. However, the discussion proposed by [15] shows that forecasting using such variables is a hard task and the results are not always as anticipated.

We now need to specify what we mean by the term 'future'. For [24], who compared ARIMA and sinusoidal representation with regression, future means hourly estimation. This research is supplemented by the multivariate regression method introduced by [25] or [26]. However, this topic has little pertinence in Pediatric ED crowding. Even if the mean square error of prediction was good enough, the bed occupancy was only predicted 4 to 12 hours downstream and concerned 1 or maybe 2 beds at most. The predictions are short-term, providing no insight into the phenomena involved, and worst of all the ED has to find solutions immediately. From a practical point of view, this does not seem feasible. If we go a step further, the predictive solutions given by [27], [28], [23] narrow it down to daily predictions. This approach seems more implementable in ED. Only a few predictive days confirm the staff perception of abnormal activity. However, the ED staff still have no insight into the trends relating to crowding as the daily patient flow varies considerably. To overcome this difficulty we looked at the problem from another angle.

Earlier in the paper we introduced the concept of normal and abnormal ED activity. We therefore consider epidemics and crowding as abnormal activity, which intuitively represents abnormal patient flow. The crowding effect is thus viewed as an outlier in the patient flow time series, namely $x(t)$, and so removing this outlier gives an idea of normal activity.

Justified by the quasi-periodicity of epidemics, we adopted a sinusoidal model expressed as

$$
\hat{x}(t)=\hat{\beta}_{0}+\hat{\beta}_{1} t+\hat{\beta}_{2} \cos \left(\frac{2 \pi}{\hat{T}_{1}} t\right)+\ldots+\hat{\beta}_{n_{p}+2} \cos \left(\frac{2 \pi}{\hat{T}_{n_{p}}} t\right) .
$$

A two-stage procedure is required. First, the set of periods $\left\{\hat{T}_{k}\right\}_{k=1}^{n_{p}}$ has to be estimated. This procedure concerns the theory of spectral analysis and is beyond the scope of this paper. To solve the problem we used a classical Fourier analysis, but many other High Resolution (HR) methods could be employed to estimate the number and the value of the different periods $\left\{\hat{T}_{k}\right\}_{k=1}^{n_{p}}$. The reader can review the work by [29], [30] and the references therein. Finally, the set $\left\{\hat{\beta}_{k}\right\}_{k=0}^{n_{p}+2}$ has to be estimated as well. This may be the key point of the model. We know that the outliers must be removed from the data collected. To do so, the methodology proposed by [18] suggests filtering the data according to the data distribution quantiles (thresholds). Some Bronchiolitis Pediatric ED flow quantiles are given in Tab.I.

\begin{tabular}{|l||c|c|c|c|c|c|}
\hline Quantile & $75 \%$ & $80 \%$ & $85 \%$ & $90 \%$ & $95 \%$ & $100 \%$ \\
\hline No. of patients & 3.00 & 3.00 & 4.00 & 6.00 & 7.55 & 16.00 \\
\hline
\end{tabular}

Distribution Quantile of Pediatric ED flow for patients diagnosed with Bronchiolitis, ChU Saint Etienne, France.

Clearly, the higher the quantile, the less outliers are removed and vice versa. The quantile selected determines the maximum normal patient flow and consequently the threshold for the early detection of crowding. From our point of view, and backed by the data distribution, we consider that outliers, and thus crowding, are events concentrated in time. Thanks to the results in section IV, which demonstrate this point more mathematically, we established the $90 \%$ quantile as the threshold for the data collected, which meant that each time the patient flow exceeded 6 , the data were clipped. We thus obtained a new Time Series defined as

$$
\forall t=1, \ldots, N \quad \tilde{x}(t)=\left\{\begin{array}{ll}
x(t) & \forall x(t) \leq 6 \\
6 & \forall x(t)>6
\end{array} .\right.
$$


The solution can be found for the set $\left\{\hat{\beta}_{k}\right\}_{k=0}^{n_{p}+2}$ using ordinary least squares

$$
\hat{\boldsymbol{\beta}}=\boldsymbol{A}^{\dagger} \tilde{\boldsymbol{x}}
$$

where

$$
\boldsymbol{A}=\left(\begin{array}{ccccc}
1 & 1 & \cos \left(\frac{2 \pi}{\hat{T}_{1}}\right) & \ldots & \cos \left(\frac{2 \pi}{\hat{T}_{n_{p}}}\right) \\
\vdots & 2 & \cos \left(\frac{2 \pi}{\hat{T}_{1}} 2\right) & \ldots & \cos \left(\frac{2 \pi}{\hat{T}_{n_{p}}} 2\right) \\
\vdots & \vdots & \vdots & & \vdots \\
1 & n & \cos \left(\frac{2 \pi}{\hat{T}_{1}} N\right) & \ldots & \cos \left(\frac{2 \pi}{\hat{T}_{n_{p}}} N\right)
\end{array}\right)
$$

with.$^{\dagger}$ the classical Moore-Penrose pseudo-inverse and $\hat{\boldsymbol{\beta}}=\left[\hat{\beta}_{0} \ldots \hat{\beta}_{n_{p}+2}\right]$. Matrix $\boldsymbol{A}$ can also be seen with respect to its rows, besides $\boldsymbol{A}=\left[\begin{array}{llll}\boldsymbol{a}_{1}^{T} & \boldsymbol{a}_{2}^{T} & \ldots & \boldsymbol{a}_{N}^{T}\end{array}\right]^{T}$. Finally, the temporal reconstruction of the model is obtained by $\hat{x}(t)=\hat{\boldsymbol{\beta}} \boldsymbol{A}^{T}$ where.$^{T}$ is the transpose operator. The error estimate

$$
\hat{\sigma}^{2}=\frac{1}{N-1} \sum_{i=1}^{N}[x(i)-\hat{x}(i)]^{2}
$$

is then used to obtain a $95 \%$ Confidence Interval written as:

$$
\begin{gathered}
\mathrm{CI}(i)=\hat{\boldsymbol{\beta}} \boldsymbol{a}_{i}^{T}+1.96 \sigma \sum_{j=1}^{N}\left[\left(\boldsymbol{A}^{T} \boldsymbol{A}\right)^{-1 / 2}\right]_{j j} a_{j i} \\
\text { for } i=1, \ldots, N .
\end{gathered}
$$

The final step of the procedure involves determining a second threshold with respect to the Confidence Interval obtained in (5). A multiple regression on the resulting Time Series is then run. This concludes the procedure to reach the estimated normal activity (normal flow) $\hat{x}(t)$ with its $95 \%$ Confidence Interval. The data collected daily from Saint Etienne Hospital for patients diagnosed with bronchiolitis and the corresponding estimated normal flow given by the Serfling approach are presented in Fig.3-(a). Four main periods have been estimated for this Time Series

$$
\left[\hat{T}_{1}=390.01, \quad \hat{T}_{2}=171.58, \quad \hat{T}_{3}=115.94, \quad \hat{T}_{4}=87.56\right]
$$

and the vector of estimated coefficients $\hat{\boldsymbol{\beta}}$ is given by

$$
[1.4922,-0.0001, \quad 1.1676, \quad 0.2688,-0.1056,0.0154] \text {. }
$$

It can be noted that the original Time Series $x(t)$ does not exhibit a linear trend. Indeed, the coefficient $\hat{\beta}_{1}$ is equal to zero. There is only a constant trend in mean, of which the integer value is approximately 2 . The threshold Confidence Interval, up to the nearest and superior integer, is given in Fig.3-(b). Based on the indicator proposed by [18], when the data collected exceeds the threshold given by the Confidence Interval, an epidemic is detected. It is therefore likely that crowding appears. This would be true if the data collected daily had a continuous trend up to the height of the epidemic. In reality, and according to the figures used hitherto, it is clear that this is not the case. So, we propose to detect a future crowding effect by improving the moment of the decision.

\section{EARLY INDEX FOR ABNORMAL ACTIVITY: USING THE KURTOSIS}

To actually solve the problem of early detection based on normal flow, the outliers need to be studied in more detail. The key lies in the analysis of the outliers or the related time series. We propose to create a new Time Series, for example $y(t)$, obtained by taking all the data values greater than the mean $\hat{x}(t)$ and of course superior to the normal activity model $x(t)$. Therefore

$$
y(t)=\left\{\begin{array}{l}
x(t)-\hat{x}(t) \quad \forall \hat{x}(t)>\overline{\hat{x}}, x(t)>\hat{x}(t) \\
0
\end{array}\right.
$$




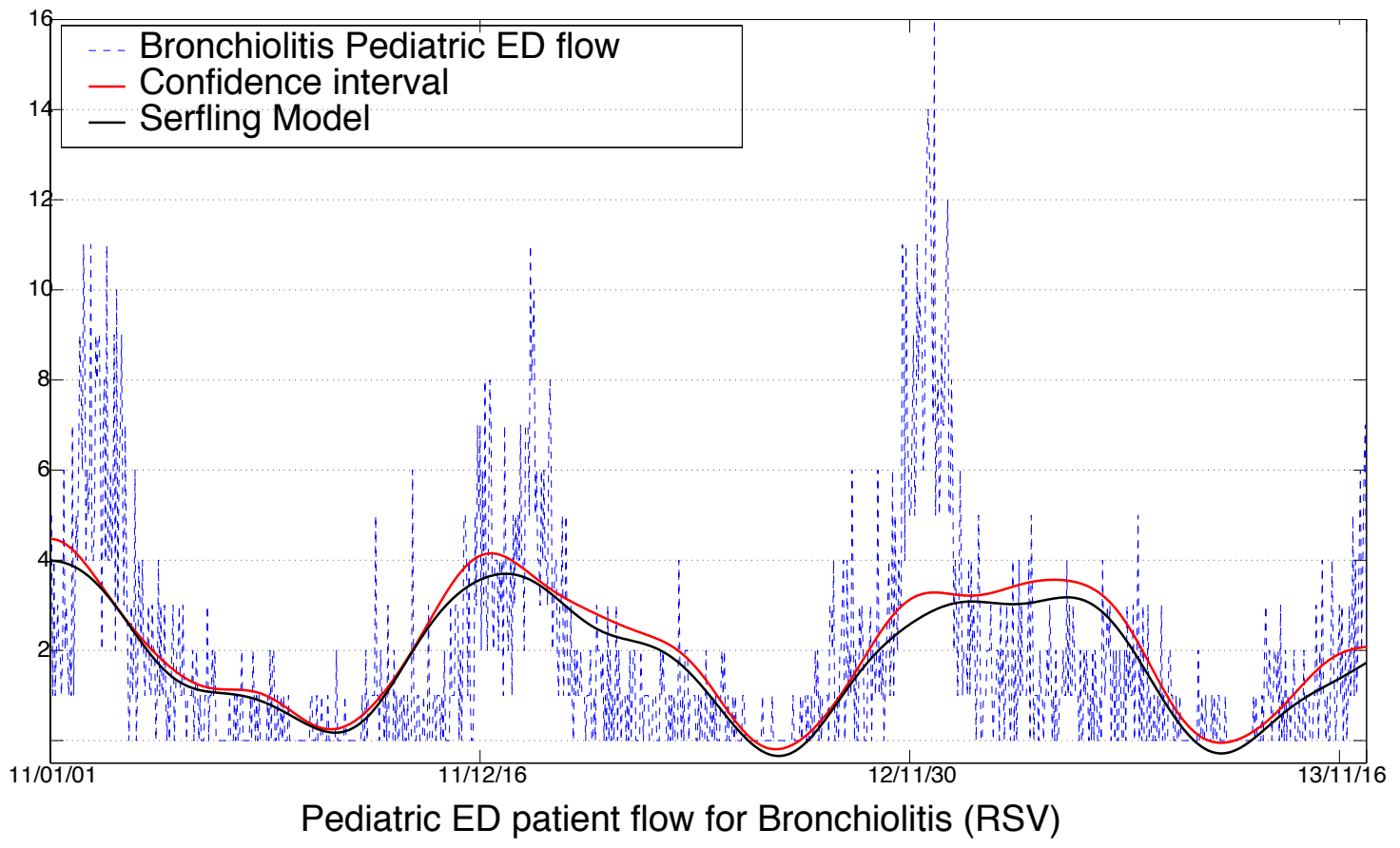

(a)

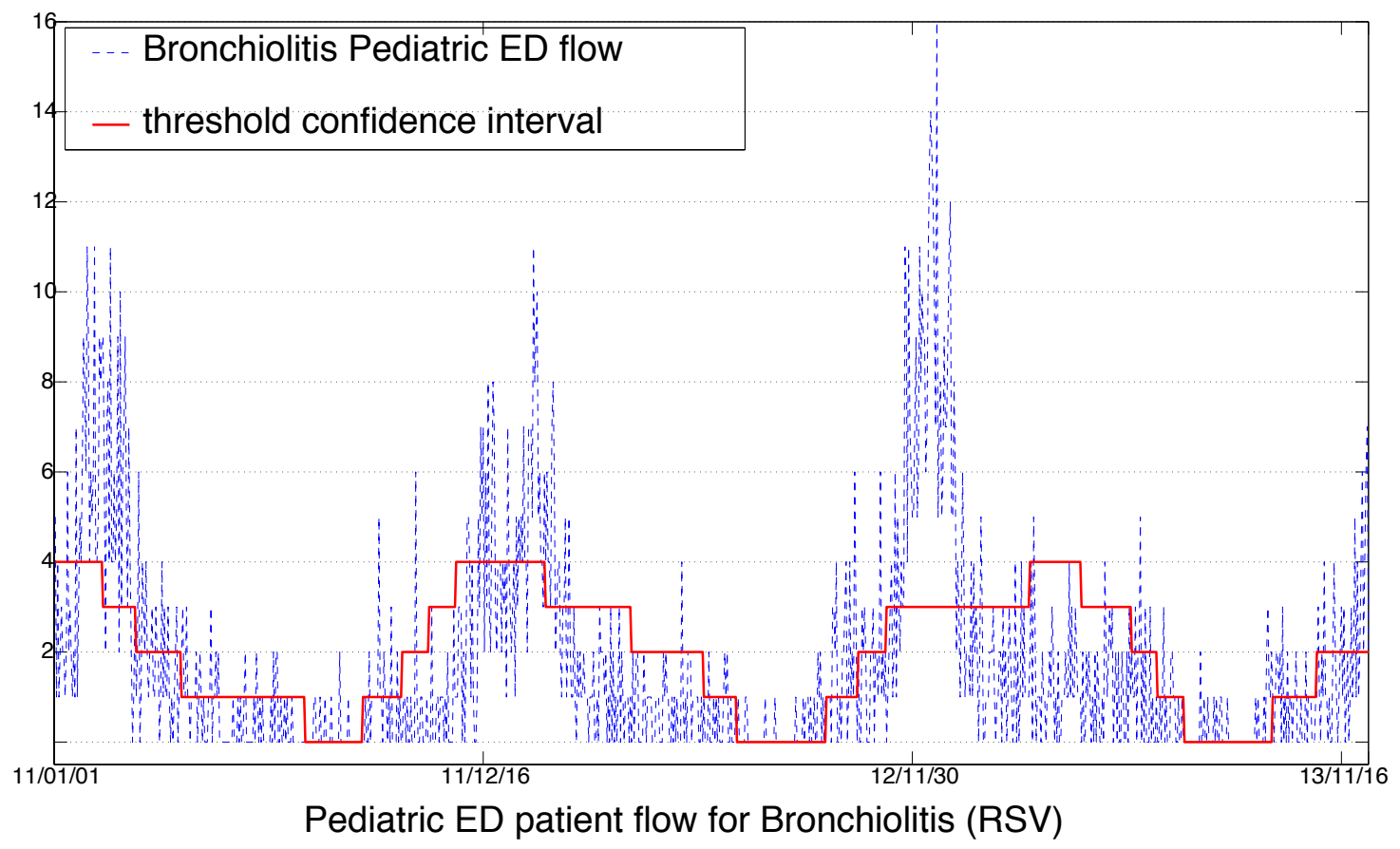

(b)

Fig. 3. (a) Daily Bronchiolitis flow and the estimated normal flow with its 95\% confidence Interval, (b) Daily Bronchiolitis flow and the 95\% threshold Confidence Interval (for integer values) for the estimated normal patient flow 
where the bar operator $(\cdot)$ defines the classical time average operator. An image of this Time Series is provided in Fig.4-(a). The series is identically null almost all the time except when epidemics occur. This confirms the previous observation that epidemics are temporally confined. By examining the shape of the estimated distribution, given by the histogram in Fig.4-(b), we can clearly see that there are very few days on which high numbers of patients arrive at the ED; this marks the exceptional nature of these days and thus an epidemic. But, by definition we have the fourth-order moment of the distribution, also known as the kurtosis, which accurately characterizes the shape of the distribution. Following the discussion proposed by [31], the kurtosis has a high value when the distribution exhibits a peak and has a heavy tail, whereas it has a low value when the distribution is more rounded and has a thinner tail tending rapidly to zero. The sample kurtosis estimator is classically given by averaging over time

$$
k_{1}=\frac{\frac{1}{N} \sum_{i=1}^{N}(x(i)-\bar{x})^{4}}{\left(\frac{1}{N} \sum_{i=1}^{N}(x(i)-\bar{x})^{2}\right)^{2}} .
$$

The Normal distribution is generally used as a reference; its kurtosis value is 3 and it is then taken to calculate the kurtosis according to the relation:

$$
k=\frac{n-1}{(n-2)(n-3)}\left((n+1) k_{1}-3(n-1)\right) .
$$

When the kurtosis value is null, the distribution of the outliers is assimilated to the Normal distribution. When it is positive, the distribution is extremely concentrated over a few values; the outliers are more uniformly distributed when it is negative. So, if we look at Fig.4-(a) once again, when attendance at the Pediatric ED is low, the values of the series are identically zeros. If the kurtosis is calculated during this period, i.e. within a specific observation window of a couple of weeks, it will have a maximum value. This is for two reasons: firstly, the constant values inevitably produce a null variance so that (7) takes an infinite value, and secondly, the empirical distribution will tend to a Dirac mass at zero. If the window now moves along time, the apparition of small values modifies the shape of the distribution, which becomes less concentrated, and so the kurtosis value quickly decreases (see Fig.5). The observation windows marked with horizontal dashed lines have voluntarily been drawn longer than they should be. The idea resides in the fact that when abnormal flow occurs, the distribution is more uniform since the number of patients grows quickly and then decreases as quickly as it increased. The kurtosis value might then be negative. So, we have a kurtosis value which is high under normal operations and decreases to zero when abnormal flow appears. This clearly defines an index which predicts crowding.

\section{Optimal Choice of QUANTILE AND Window SizE}

Obtaining the early kurtosis index is largely parameter dependent. To detect abnormal flow early, normal flow needs to be characterized first. This is the subject of section II in which we discussed the particular choice of quantile percentage for the present section. In conjunction with this parameter, the observation time should also have a significant effect on detection. To solve this $2 \mathrm{D}$ issue, we propose to maximize the following criterion in both the quantile percentage, materialized by the unknown $q$, and the window size associated with the unknown $w$. This criterion is written as

$$
\begin{aligned}
& (\hat{q}, \hat{w})=\arg \max \mathcal{F}(q, w) \\
& \text { s.t. }\left|\mathcal{I}_{k} \stackrel{q, w}{-} \mathfrak{I}_{E}\right| \leq D
\end{aligned}
$$

with

$$
\mathcal{F}(q, w)=\left\|\mathcal{I}_{\boldsymbol{k}}(q, w)-\mathcal{I}_{\boldsymbol{E}}\right\|_{2}^{2}-\boldsymbol{\lambda}\left|n_{k}(q, w)-n_{E}\right|^{2} .
$$

The vectors $\mathcal{I}_{k}(q, w)$ and $\mathcal{I}_{\boldsymbol{E}}$ are the vectors of the dates when the kurtosis index is negative (early date for detection of abnormal flow) and the dates related to the maximum number of patients for each epidemic (date of maximum flow), respectively. So, we sought to maximize early detection with respect to the epidemic peak. We nevertheless imposed a maximum number of days $\mathrm{D}$ for detection. This means that we do not accept detection dates that are more than $\mathrm{D}$ days before the epidemic peak. According to our data, an epidemic lasts about 60 days and is roughly symmetric each year. So, we fixed $D=30$ days. Now, if the early kurtosis index never crosses zero, some dates will be lacking. Each missing date is replaced by its corresponding date of maximum flow. This leads 


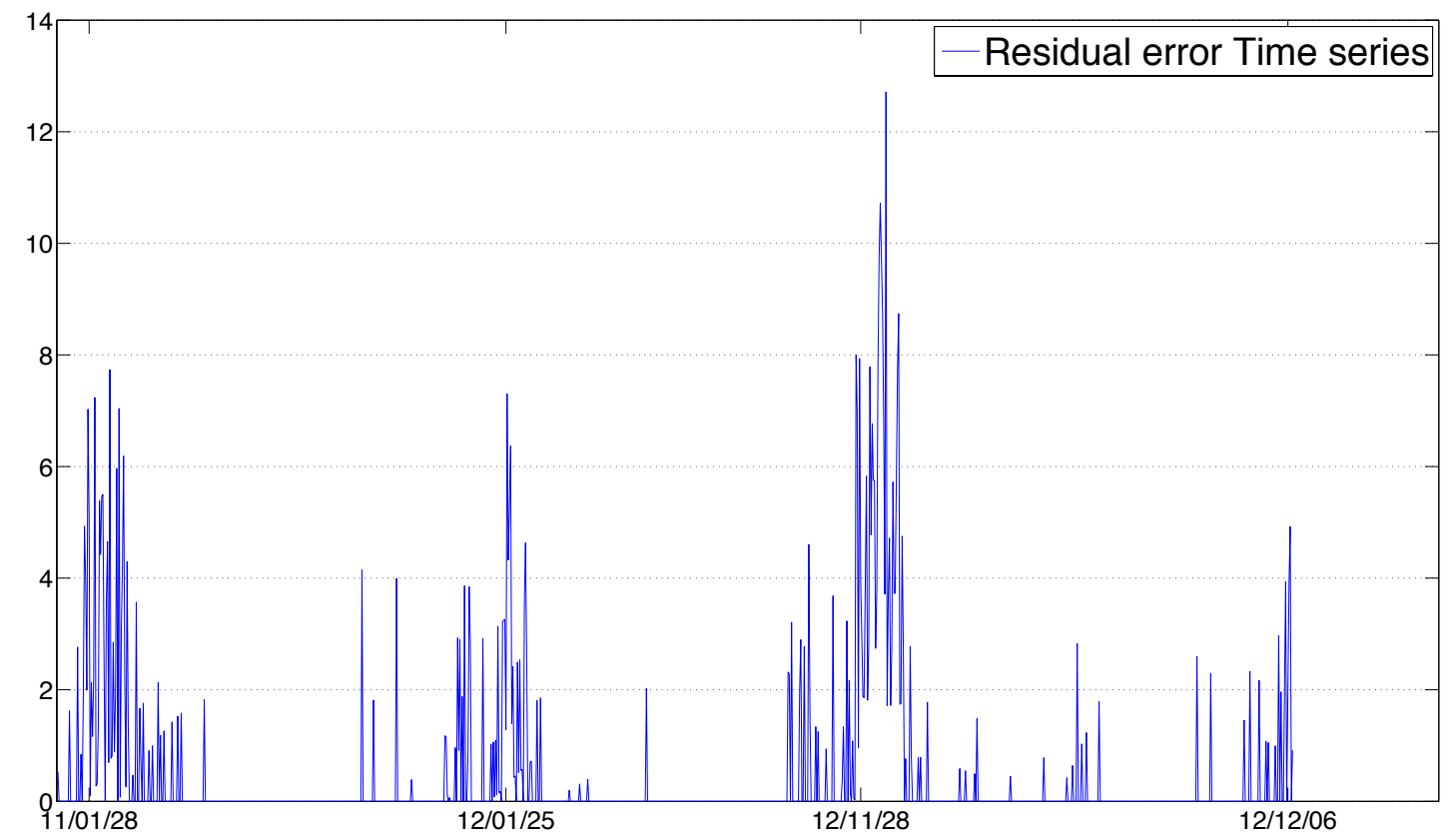

(a)

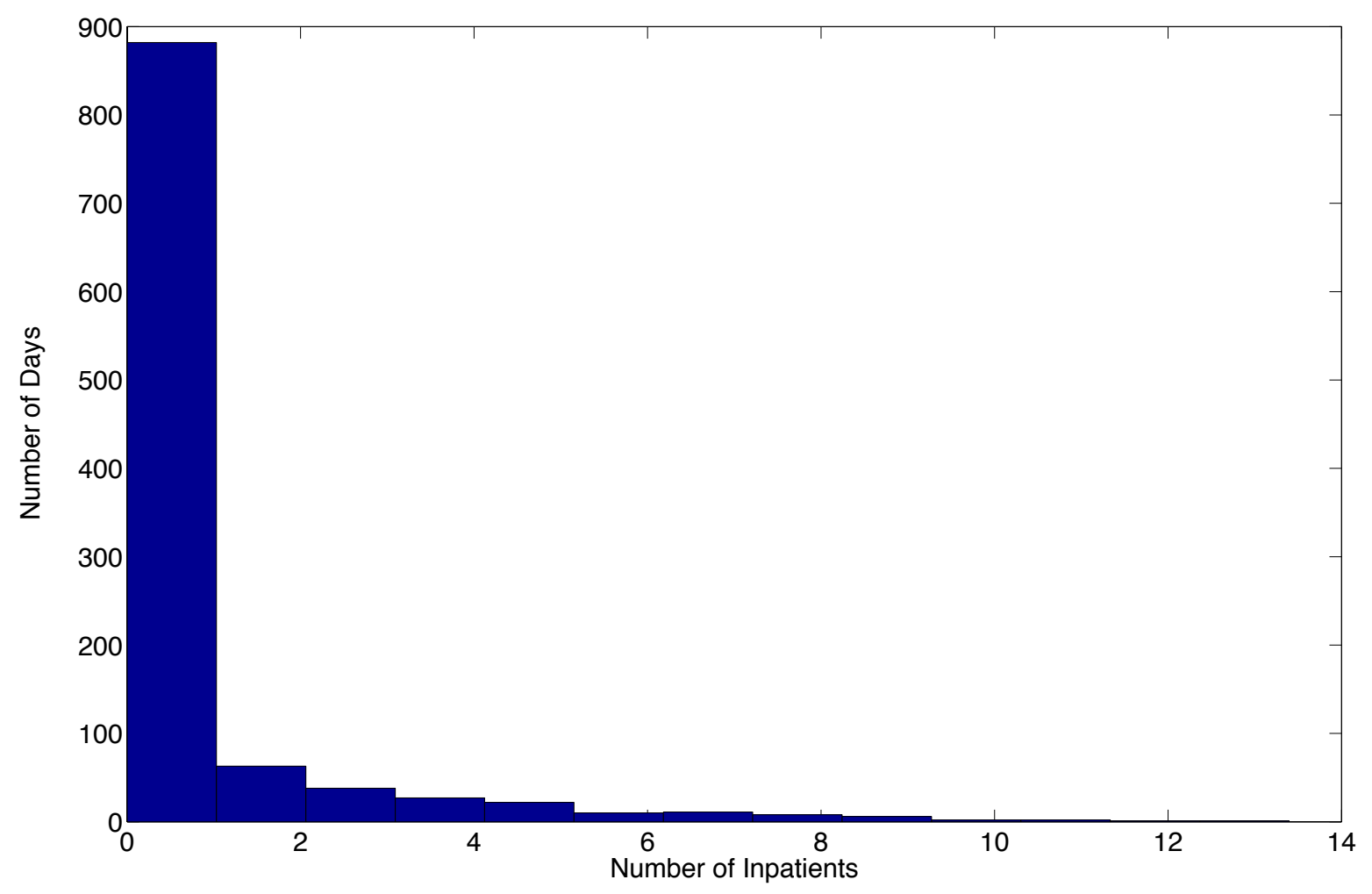

(b)

Fig. 4. (a) Outlier Time Series, (b) histogram of the Outlier Time Series 


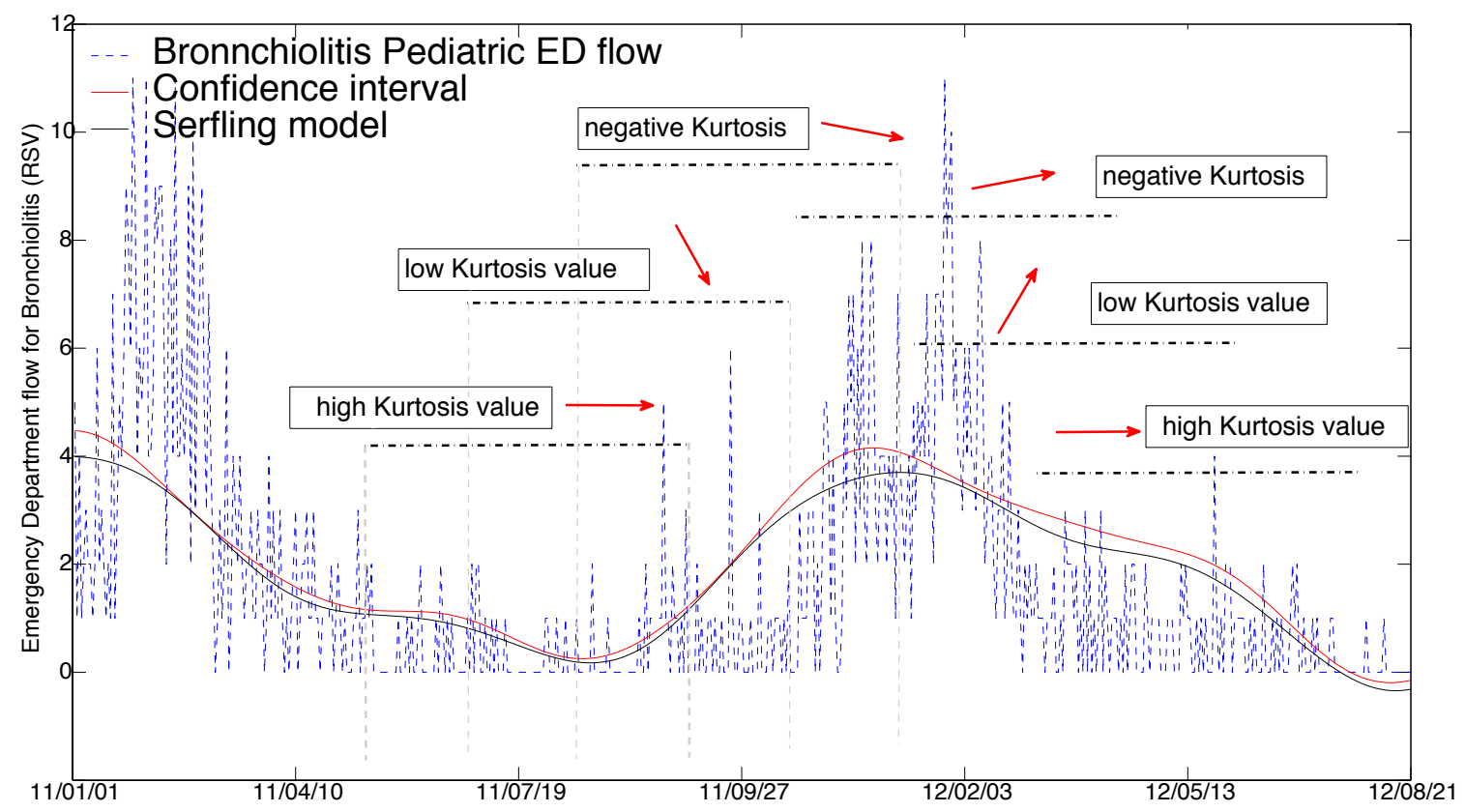

Fig. 5. Evolution of the kurtosis according to the example, the arrows correspond to the variations in the kurtosis calculated, for illustrative purposes and understanding over a large window of about 4 months.

to the penalization term in equation (10), which penalizes the function $\mathcal{F}(q, w)$ by a factor $\boldsymbol{\lambda}$ when the number of detections $n_{k}(q, w)$ is not equal to the number $n_{E}$ of epidemics found. Parameter $\boldsymbol{\lambda}$ takes two values

$$
\boldsymbol{\lambda}=\left\{\begin{array}{lll}
10 & \forall & n_{k}(q, w) \geq n_{E} \\
200 & \forall & n_{k}(q, w)<n_{E}
\end{array}\right.
$$

so that non-detection is heavily penalized with regards to over detection.

We have computed the objective function $\mathcal{F}(q, w)$ for the flow of patients diagnosed with Bronchiolitis. A representation of this function is given in Fig.6. By analyzing this figure, it can be observed that the function seems less sensitive to the choice of quantile than to the choice of observation window. However, it seems that high quantiles are recommended and more relevant. Finally, the optimum was 6 patients for a 90\% quantile with an observation window of 14 days. These results explain the choice of parameters in sections II and V.

\section{Illustration of the Pediatric ED data from Saint Etienne Teaching Hospital}

This section is dedicated to illustrating the relevance of the method for announcing abnormal activity using real data collected from Saint Etienne Teaching Hospital.

An Excel application using VBA was developed for the method proposed and was given to the Pediatric ED in midJuly 2013 for testing. Fig.7 is a snapshot of the application given to the physicians. The Pediatric ED Bronchiolitis flow is marked by a dashed line; the Serfling model and the kurtosis value were calculated over a sliding window of 14 days. We reiterate that each time the kurtosis goes below zero, implying that the kurtosis moves from a positive to a negative value, abnormal activity, most likely an epidemic, is detected.

Firstly, the Serfling representation models the ED activity well. Indeed, on the extreme right-hand side of the plot, we can see that the Pediatric ED activity increases for patients diagnosed with Bronchiolitis at almost the same rate as the Serfling model. The second important point resides in the analysis of the kurtosis plot. Over the three years examined, there were five exceedences; the kurtosis moved below zero at least three times when the epidemics started and for two virus-dependent phenomena. A sixth exceedence was actually observed on around 6th December 2013, which indicated the start of a Bronchiolitis epidemic. This part of the plot is totally experimental; when the article was being written, Saint Etienne Teaching Hospital was aware that crowding would occur in the next couple 


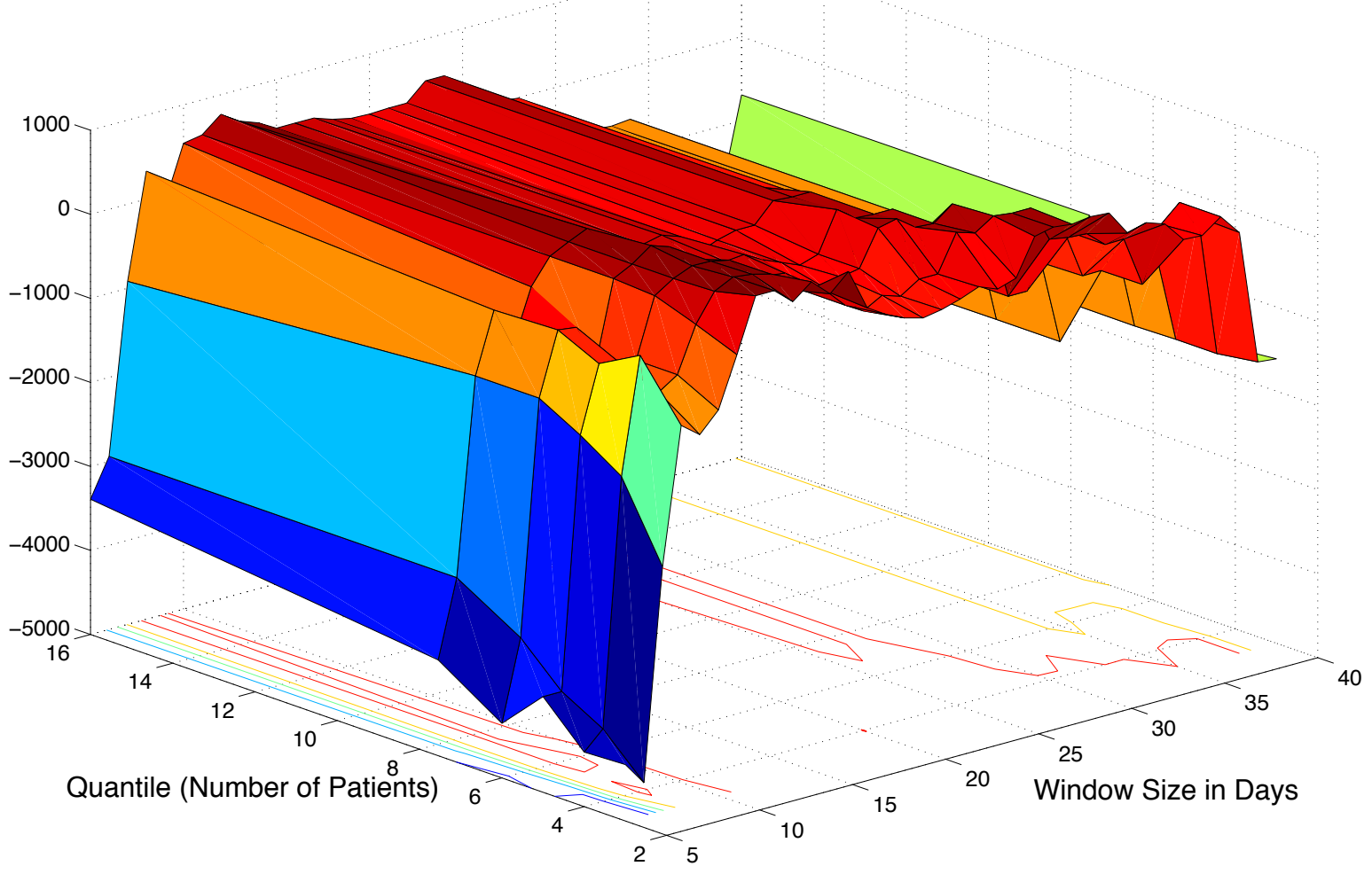

Fig. 6. Representation of criteria (10) for quantiles varying within the range $[3,16]$ and observation times varying within the range $[5,40]$ days. The maximum was observed for the $90 \%$ quantile (6 patients) and an observation window of 14 days.

of weeks if the emergency plan was not requested. This was the observation in real time of the onset of an epidemic. We will now illustrate how the application improved decision-making in the pediatric ED in Saint Etienne Hospital and how crowding could be minimized. As an example, we will look at the case of the last epidemic (at the time when the paper was being written), which took place between November 2012 and February 2013. The following discussion is supported by Fig.8 where the progress of operations to reduce crowding are reported in chronological order. At the time, the Pediatric ED had the application presented above at its disposal. As we can see in Fig.8, the ED requested an emergency plan to reduce crowding on 12th December 2012, but the plan was only accepted and activated on 18th December 2012. By establishing a parallel between this date and the corresponding Pediatric ED flow, we can see that the epidemic was at its height. This observation is reinforced by the bed occupancy figures given in Fig.1, where straightforwardly the derivative is negative and the Hospital needed less and less beds. The decision came too late. If we now examine the early index proposed, the start of abnormal activity (epidemic in perspective) was detected on 28th November 2012, almost three weeks before the activation of the emergency plan. By doing the same parallel with beds occupancy of Fig.1, we understand that the worst could have been avoided. Since mid-July 2013, the Pediatric ED in Saint Etienne Teaching Hospital has the application which provides the early index for crowding detection proposed in this paper. Thanks to this early index, abnormal activity was detected around 6th December 2013. On this date the head of the Pediatric ED requested and obtained the authorization for the activation of the emergency plan.

\section{CONCLUSION}

When the number of patients who need special care exceeds the capacity of the ED, crowding inevitably occurs. The effects of crowding have been widely documented throughout the world and are the same wherever the ED. 


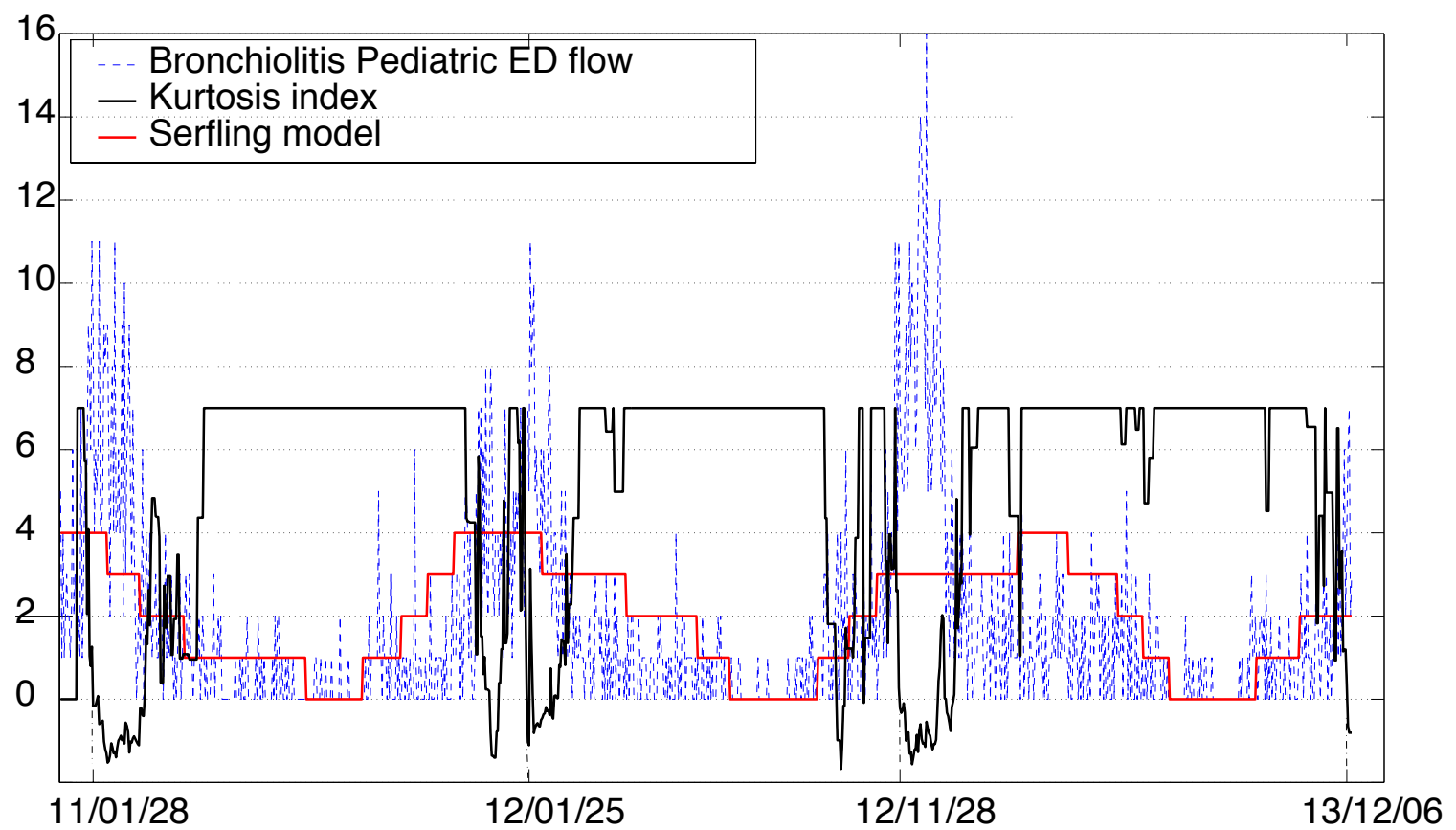

Fig. 7. Application of Sliding kurtosis on Saint Etienne Bronchiolitis Pediatric ED flow data.

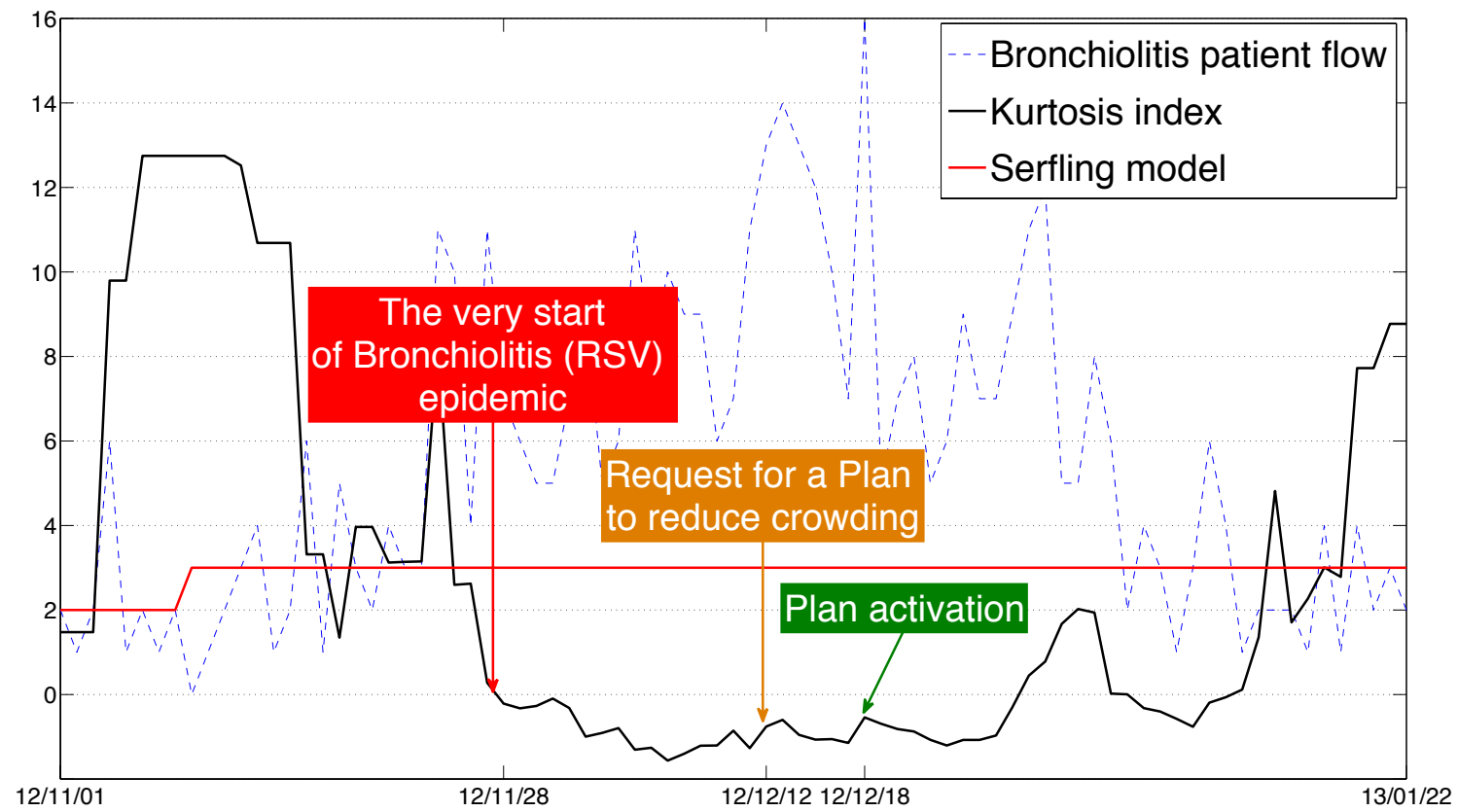

Fig. 8. Real time decisions of Saint Etienne Hospital in orange (middle) and green (right), and instant detection of abnormal activity in red (left) from the Bronchiolitis Pediatric ED flow of Nov. 2012 to Feb. 2013. 
It is most certainly for these reasons that over the past few decades the literature has focused on methodologies to prevent crowding. One possible way of solving the issue is to propose a patient flow forecasting model. In this regard, there is much research in the literature concerning weekly predictions and the proposed methods are not really applicable to daily predictions. Consequently, we have chosen to tackle the problem by considering that crowding is closely related to abnormal patient flow. So, the prevention of crowding resides firstly in providing a model for normal daily patient flow and secondly, in proposing an early index which detects abnormal visits. In this paper, we proposed a multi-period Serfling-based model for daily patient flow. Then we built a Time Series obtained from the difference between the real data and the model. Finally, the kurtosis calculated over a time window within this Time Series was computed as it detects abnormally high patient numbers at the ED. The methodology presented previously is parameter dependent. Consequently, we provided a criterion which gives the optimal parameters to be chosen with respect to the observations. This early index has been applied to real data collected from the Pediatric ED in Saint Etienne Teaching Hospital (CHU Saint Etienne), France, and has shown very good performances. This index may have enabled a gain of almost three weeks for the Bronchiolitis epidemic in 2012 and in 2013 enabled an emergency plan to be activated early in December, almost three weeks before the height of the epidemic. The software, which integrates the methodology proposed in this paper, is also being deployed in different Pediatric ED in Rhone-Alpes, France. Our future work will concern the outlier Time Series as it is essential for the procedure and for the Hospitals to plan their activity.

\section{ACKNOWLEDGEMENTS}

The authors are very grateful to Mr. Bernadac, CHU Saint Etienne for his full implication and his work in making the data easy to process. We would also like to thank Mr Colmant, ARS Rhone-Alpes, for his help in deploying the software.

\section{REFERENCES}

[1] N. Hoot, L. LeBlanc, I. Jones, S. Levin, C. Zhou, C. Gadd, and D. Aronsky, "Forecasting emergency department crowding: A discrete event simulation," Annals of emergency medicine, vol. 52, no. 2, pp. 116-125, 2008.

[2] N. Hoot and D. Aronsky, "Systematic review of emergency department crowding: Causes, effects, and solutions," Annals of Emergency Medicine, vol. 52, no. 2, pp. 126 - 136.e1, 2008.

[3] G. Hung, S. Whitehouse, C. ONeill, A. Gray, and N. Kissoon, "Computer modeling of patient flow in a pediatric emergency department using discrete event simulation." Pediatric emergency care, vol. 23, no. 1, pp. 5-10, Jan. 2007.

[4] A. Arenas, G. Gonzalez-Parra, and J. Morano, "Stochastic modeling of the transmission of respiratory syncytial virus (rsv) in the region of valencia, spain," Biosystems, vol. 96, no. 3, pp. 206-212, 2009.

[5] D. R. Eitel, S. Rudkin, M. Malvehy, J. Killeen, and J. Pines, "Improving service quality by understanding emergency department flow: A white paper and position statement prepared for the american academy of emergency medicine," The Journal of Emergency Medicine, vol. 38, no. 1, pp. 70-79, 2010.

[6] J. Peck, S. Gaehde, D. Nightingale, D. Gelman, D. Huckins, M. Lemons, E. Dickson, and J. Benneyan, "Generalizability of a simple approach for predicting hospital admission from an emergency department." Academic emergency medicine : official journal of the Society for Academic Emergency Medicine, vol. 20, no. 11, 2013.

[7] R. Hall, D. Belson, P. Murali, and M. Dessouky, "Patient flow: Reducing delay in healthcare delivery," ser. International Series in Operations Research \& Management Science, R. Hall, Ed. Springer US, 2006, vol. 91, pp. 1-44.

[8] A. Stang, D. McGillivray, M. Bhatt, A. Colacone, N. Soucy, R. L $\sqrt{ }$ C ger, and M. Afilalo, "Markers of overcrowding in a pediatric emergency department," Academic Emergency Medicine, vol. 17, no. 2, pp. 151-156, 2010.

[9] J. Lyon, G. Stoddard, D. Ferguson, M. Caravati, A. Kaczmarek, G. Thompson, K. Hegmann, and C. Hegmann, "An every other year cyclic epidemic of infants hospitalized with respiratory syncytial virus," Pediatrics, vol. 97, no. 1, pp. 152-153, 1996.

[10] C. Panozzo, A. Fowlkes, and L. Anderson, "Variation in timing of respiratory syncytial virus outbreaks: lessons from national surveillance," Pediatric Infect. Dis. Journal, vol. 26, no. 11 Suppl, pp. S41-45, 2007.

[11] M. Jose and R. Bishop, Scaling properties and symmetrical patterns in the epidemiology of rotavirus infection. London, ROYAUMEUNI: Royal Society of London : Biological Sciences, 2003, vol. 358, no. 1438.

[12] C.-F. Chen, W.-H. Ho, H.-Y. Chou, S.-M. Yang, I.-T. Chen, and H.-Y. Shi, "Long-term prediction of emergency department revenue and visitor volume using autoregressive integrated moving average model," Computational and Mathematical Methods in Medicine, vol. 2011, p. 7, 2011.

[13] Suhartono, "Time series forecasting by using seasonal autoregressive integrated moving average: subset, multiplicative or additive model." Journal of Math. and Stat., vol. 7, no. 1, pp. 20-27, 2011.

[14] M. Capistran, M. Moreles, and B. Lara, "Parameter estimation of some epidemic models. the case of recurrent epidemics caused by respiratory syncytial virus," Bull Math Biol, vol. 71, no. 8, pp. 1890-1901, 2009.

[15] N. Walton, M. Poynton, P. Gesteland, C. Maloney, C. Staes, and J. Facelli, "Predicting the start week of respiratory syncytial virus outbreaks using real time weather variables," BMC Medical Informatics and Decision Making, vol. 10, no. 1, 2010.

[16] L. Simonsen, C. Viboud, R. Taylor, and M. Miller, "The epidemiology of influenza and its control," in Influenza Vaccines for the Future, ser. Birkh $\sqrt{ }$ user Advances in Infectious Diseases, R. Rappuoli and G. Del Giudice, Eds. Springer Basel, 2011 , pp. $27-54$. 
[17] W. Thompson, L. Comanor, and D. Shay, "Epidemiology of seasonal influenza: Use of surveillance data and statistical models to estimate the burden of disease," Journal of Infectious Diseases, vol. 194, no. Supplement 2, pp. S82-S91, 2006.

[18] R. Serfling, "Methods for current statistical analysis of excess pneumonia-influenza deaths," Public health reports, vol. 78, no. 6, pp. 494-506, Jun. 1963.

[19] I. Soyiri and D. Reidpath, "Evolving forecasting classifications and applications in health forecasting." International journal of general medicine, vol. 5, 2012.

[20] J. Wiler, R. Griffey, and T. Olsen, "Review of modeling approaches for emergency department patient flow and crowding research," Academic Emergency Medicine, vol. 18, no. 12, pp. 1371-1379, 2011.

[21] J. Peck, "Using prediction to facilitate patient flow in a health care delivery chain," Ph.D. dissertation, MIT, 2013.

[22] A. Zlotnik, J.-M. Montero-Mart $\sqrt{ } \neq$ nez, and A. Gallardo-Antol $\sqrt{ } \neq \mathrm{n}$, "A comparison of multivariate sarima and svm models for emergency department admission prediction.” in HEALTHINF, D. Stacey, J. Sol $\sqrt{ }$ C -Casals, A. L. N. Fred, and H. Gamboa, Eds., 2013, pp. 245-249.

[23] I. Marcilio, S. Hajat, and N. Gouveia, "Forecasting daily emergency department visits using calendar variables and ambient temperature readings." Academic emergency medicine : official journal of the Society for Academic Emergency Medicine, vol. 20, no. 8, 2013.

[24] L. Schweigler, J. Desmond, M. McCarthy, K. Bukowski, E. Ionides, and J. Younger, "Forecasting models of emergency department crowding," Academic Emergency Medicine, vol. 16, no. 4, pp. 301-308, 2009.

[25] S. Jones, R. Evans, T. Allen, A. Thomas, P. Haug, S. Welch, and G. Snow, "A multivariate time series approach to modeling and forecasting demand in the emergency department," Journal of Biomedical Informatics, vol. 42, no. 1, pp. 123-139, 2009.

[26] R. Rosychuk, T. Klassen, D. Voaklander, A. Senthilselvan, and B. Rowe, "Seasonality patterns in croup presentations to emergency departments in alberta, canada: a time series analysis." Pediatric emergency care, vol. 27, no. 4, 2011.

[27] G. Abraham, G. Byrnes, and C. Bain, "Short-term forecasting of emergency inpatient flow," IEEE Transactions on Information Technology in Biomedicine, vol. 13, no. 3, pp. 380-388, 2009.

[28] J. Peck, J. Benneyan, D. Nightingale, and S. Gaehde, "Predicting emergency department inpatient admissions to improve same-day patient flow," Academic Emergency Medicine, vol. 19, no. 9, pp. 1045-1054, 2012.

[29] P. Stoica, P. H $\sqrt{ } \S$ ndel, and T. S $\sqrt{ } \partial \operatorname{derstr} \sqrt{ } \partial \mathrm{m}$, “Approximate Maximum Likelihood Frequency Estimation,” Automatica, vol. 30, no. 1, pp. 131-145, Jan. 1994.

[30] M. Christensen, A. Jakobsson, and S. Jensen, "Joint high-resolution fundamental frequency and order estimation," IEEE Transactions on Audio, Speech, and Language Processing, vol. 15, no. 5, pp. 1635-1644, July 2007.

[31] L. DeCarlo, "On the meaning and use of kurtosis," Psychological Methods, vol. 2, no. 3, pp. 292-307, Sep. 1997. 